\title{
Evaluation and Analysis of the Impact of Airport Delays
}

\author{
Fen Zhou, Guosong Jiang, Zhengwu Lu, and Qingdong Wang $\mathbb{C}$ \\ School of Computer Science and Technology, Huanggang Normal University, HuangGang Hubei 438000, China \\ Correspondence should be addressed to Qingdong Wang; wangqingdong@hgnu.edu.cn
}

Received 18 December 2021; Revised 12 January 2022; Accepted 23 January 2022; Published 24 February 2022

Academic Editor: Baiyuan Ding

Copyright (c) 2022 Fen Zhou et al. This is an open access article distributed under the Creative Commons Attribution License, which permits unrestricted use, distribution, and reproduction in any medium, provided the original work is properly cited.

To characterize the real dynamic process of delay propagation between airports and to understand the mechanism of delay propagation from a global perspective, this paper establishes a network of delay propagation relationships between airports based on causal analysis and conducts an example analysis. First, the flight operating status data processing is used to obtain the arrival delay time series of each airport. Then, the delay propagation relationship between airports is analyzed in pairs through the causal analysis method to obtain the delay propagation relationship network between all airports. Finally, the complex network theory and related indicators are used. The network is analyzed, and these analysis results can provide theoretical support for the formulation of delay propagation mitigation measures.

\section{Introduction}

With the rapid development of today's air transport industry [1], the number of flights operated by airlines has increased significantly. The problem of declining punctuality has followed, and flight delays have gradually emerged. As a result, the air transport industry will suffer substantial economic losses every year [2] and even endanger the safety of passengers, aircraft, and airports [3]. Therefore, flight delays have become a significant challenge facing the air transportation system [4]. The upstream and downstream flights share aviation resources, such as aircraft, crew, and airport ground resources. Therefore, when the upstream flight is delayed, the downstream flight will also be delayed with significant probability. This phenomenon is called delay propagation $[5,6]$. If there is no reasonable method to control the propagation of delays, the spread of delays will continue to expand $[7,8]$. At the same time, due to the current development of the air transportation industry, the aviation operation scene has become very complicated, and flights will be delayed due to varying degrees of influence from different sources at the same time. Therefore, the research on the propagation mechanism of flight delays is essential and challenging.

So far, there has been a lot of research in flight delay propagation. For example, Beatty et al. [9] put forward the concept of delay multiplier to quantify delay propagation by analyzing an airline's flight status table. The delay propagation analysis of multiple flights at a single airport is carried out. These methods mainly study the local dynamics of some flights, a single airport, or an airline [10-14]. Only a small number of people research the air transportation system level from the perspective of the entire aviation network. Therefore, in the field of flight delay propagation, there is still a lack of away from the perspective of the whole aviation network that can accurately dig out the causal relationship of flight delays from the current highly complex data: the delay propagation relationship between airports. Obtaining the flight delay propagation relationship is helpful to understand the delay propagation mode and law between airports, provide theoretical support for formulating control delay propagation measures, and have guiding significance for the improvement of flight delays.

\section{Related Work}

The academic literature on flight delays can be divided into three categories:

(1) Statistical models that explore the impact of various components of travel time 
(2) Econometric models that analyze the economic drivers of flight delays

(3) Operational management models

Among them, the impact of air transportation delays on operations is investigated. Due to the highly random nature of air transportation, different aspects of flight scheduling problems have been explored in the past. Some researchers have developed statistical models for predicting the other components of air travel time. In the econometric model, the influence of various factors on the onset and progress of propagation delay is quantified. Either way, it is necessary to analyze the delay propagation process from a broader and network-based perspective because the flight schedules operated by airlines and airports are oriented towards network performance optimization. Although progress has been made in understanding the spread of flight delays [15-20], few studies have investigated the space of delays by considering the interdependence of delays. With the continuous development of time-series analysis, much progress has been made in various fields. The time-series dependency analysis [21] and medical applications of complex human body systems (such as brain-computer interface [22-26], epilepsy [27, 28], sleep staging [29-34]). Since the air transportation system is also typically large and complex, the mechanism of delay propagation has not been fully understood, especially the interdependence between different airports. Therefore, understanding the spread of flight delays is a complex problem, and few studies have studied the space of delays by considering the interdependence of delay time series. Based on the dependence of delays and using network graph theory similar to [35-40], we evaluate and analyze the airport flight delay network.

\section{Network Analysis Evaluation Index}

We use a flight delay propagation relationship discovery algorithm based on transfer entropy. This algorithm is used to discover the delay propagation relationship between two airports. Due to the large number of airports and complex interactions, the characteristics of delayed propagation cannot be understood from only the information at the level of a single airport. The complex network theory and related indicators provide a suitable method for studying air transportation systems. Therefore, network-level analysis is used to capture the global structure of functional interaction.

$A_{i j=}\left(a_{i j}\right)_{N * N}$ is the adjacency matrix of the sample network. $a_{i j}=1$ if and only if there is an edge from one node $i$ to another node $j$; otherwise, $a_{i j}=0$. In this sample network, the total number of airports is $\mathrm{N}$, and the total number of edges is $M=\sum_{i, j=1}^{N} a_{i j}=12$. Later, some practical topological structures were introduced to help analyze the delay propagation.

3.1. Degree. The degree of an airport reflects the number of airports with which it has a delayed propagation relationship. In the directed network, the in-degree $k_{i}^{i n}=\sum_{j=1}^{N} a_{j i}$ and the out-degree $k_{i}^{\text {out }}=\sum_{j=1}^{N} a_{i j}$ of airport $i$, respectively, represent the delay from airport $j$ propagate to airport $i$ and delays propagate from airport $i$ to airport $j$. The total degree of airport $i$ is $k_{i}=k_{i}^{\text {in }}+k_{i}^{\text {out }}$.

3.2. Reciprocity Parameter. The reciprocity parameter reflects the two-way nature of delay propagation between airport pairs. Reciprocity means that airport $i$ affects airport $j$, and airport $j$ also affects airport $i\left(a_{i j}=a_{j i}=1\right)$. The parameter $R$ is used to evaluate the symmetry of the directed network. It is defined as follows:

$$
R=\frac{\sum_{i \neq j}^{N}\left(a_{i j}-\bar{a}\right)\left(a_{j i}-\bar{a}\right)}{\sum_{i \neq j}^{N}\left(a_{i j}-\bar{a}\right)^{2}} .
$$

Here, $\bar{a}=\sum_{i \neq j}^{N} a_{i j} / N(N-1)$. The maximum $R$ is 1 , which means that the links between all airport pairs are bidirectional. The larger the $R$ value, the more symmetrical the network.

3.3. Link Density. Link density reflects the proportion of active links in the total number of potential links, that is, the proportion of airport pairs that influence all airport pairs. The calculation formula is as follows:

$$
l_{\mathrm{d}}=\frac{\sum_{i \neq j}^{N} a_{i j}}{N^{2}}=\frac{M}{N^{2}} .
$$

The higher the $l_{\mathrm{d}}$, the tighter the network connection, the easier it is for the delay to spread through it and the less likely it is to stop the delay from spreading.

3.4. Transitivity. Transitivity measures the existence of triangles in the network. Mathematically defined as the relationship between the number of triangles ( 3 nodes, each pair of nodes have edges) $N_{-} \Delta$ and the number of connected triples N_3 in the network, the formula is as follows:

$$
T=\frac{3 N_{\Delta}}{N_{3}} \text {. }
$$

Here, $\quad N_{\Delta}=\sum_{k>i>j} a_{i j} a_{i k} a_{j k}, \quad N_{3}=\sum_{k>i>j}\left(a_{i j} a_{i k}+\right.$ $\left.a_{j i} a_{j k}+a_{k i} a_{k j}\right)$. The existence of a large number of triangles indicates that the groups of three airports are closely connected, so the delay generated in any one of them can easily spread to other airports.

3.5. Assortativity. The coordination coefficient reflects whether nodes with similar degree values tend to be connected. It can be expressed by the conditional probability, that is, the probability of an airport with degree $k$ and an airport with the degree of influence. The formula is as follows:

$$
\frac{1}{M} \sum_{j>i} \frac{1}{2}\left(k_{i}+k_{j}\right) a_{i j}
$$

A positive co-match coefficient value indicates that airports with large degrees, in general, tend to airports with high connectivity, and the network is said to be co-matched; 
a negative co-match coefficient value indicates that airports with large degrees, in general, tend to airports with low connectivity, which is said that the network is heterogeneous.

3.6. Efficiency. The efficiency of the network reflects how easy the delay is to spread between the two airports, that is, how many intermediate nodes the delay has to pass from a node to reach the target node. The formula is as follows:

$$
E=\frac{1}{n(n-1)} \sum_{i \neq j} \frac{1}{d_{i j}} \text {. }
$$

Here, $d_{\mathrm{ij}}$ is the distance (or the number of hops) between nodes $i$ and $j$.

3.7. Clustering Coefficient. The clustering coefficient reflects the inherent clustering trend of airports. The aggregation coefficient of an airport is the proportion of adjacent airports (airports with delayed propagation links with other airports) that have direct delay propagation links (that is, the number of triangles in the network). For the network, the overall clustering coefficient is calculated as follows:

$$
\begin{gathered}
C^{\mathrm{D}}=\frac{1}{n} \sum_{i=1}^{n} \frac{(1 / 2) \sum_{j} \sum_{h}\left(a_{i j}+a_{j i}\right)\left(a_{i h}+a_{h i}\right)\left(a_{j h}+a_{h j}\right)}{\left[d_{\mathrm{i}}^{\mathrm{dot}}\left(d_{\mathrm{i}}^{\mathrm{dot}}-1\right)-2 d_{\mathrm{i}}^{\leftrightarrow}\right]} . \\
\text { Here, } d_{i}^{\text {dot }}=\sum_{j \neq i} a_{j i}+\sum_{j \neq i} a_{i j} \text { and } d_{i}^{\leftrightarrow}=\sum_{j \neq i} a_{i j} a_{j i} .
\end{gathered}
$$

3.8. Largest Connected Cluster. The largest connected cluster reflects the degree of delayed propagation. The largest related group is through a set of interconnected airports. To represent the area of delayed propagation, we set a baseline for the connected cluster. Only if an airport affects enough airports will it be added to the connected group; it is greater than a certain threshold.

To measure the similarity of the largest connected clusters of different networks, the Jaccard index is introduced, which is defined as $J=|A \cap B| /|A \cup B|$, where $\mathrm{A}$ and $\mathrm{B}$ are both finite sets composed of airport members in the largest connected cluster. If the airport sets are the same, then $J=1$; if they are completely different, then $J=0$.

3.9. Community. The community assesses whether airport delay propagation can be divided into multiple subregions. Each subregion has dense delay propagation links inside the airport and sparse delay propagation links with airports outside the subregion. In addition, the modular $Q_{d}$ is used to measure the strength of dividing the network into multiple communities. The calculation formula of $Q_{d}$ is as follows:

$$
Q_{\mathrm{d}}=\frac{1}{M} \sum_{i j}\left(A_{\mathrm{ij}}-\frac{k_{i}^{\mathrm{out}} k_{j}^{\mathrm{in}}}{M}\right) \delta\left(c_{i}, c_{j}\right) .
$$

Here, if airport $i$ and airport $j$ are in the same community, the $\delta$ function outputs 1 ; otherwise, it outputs $0 . M$ represents the total number of edges.
3.10. Network Motifs. The network motif reflects the local relationship pattern between any three airports. The three airport clusters have similar relationship patterns. An essential tool for evaluating the significance level of the motif is Z-score, which is defined as follows:

$$
Z\left(G^{\prime}\right)=\frac{F_{G}\left(G^{\prime}\right)-\mu_{R}\left(G^{\prime}\right)}{\sigma_{R}\left(G^{\prime}\right)},
$$

where $F_{G}\left(G^{\prime}\right)$ represents the frequency of $G^{\prime}$ in the network G. $\mu_{R}\left(G^{\prime}\right)$ and $\sigma_{R}\left(G^{\prime}\right)$, respectively, represent the frequency average and standard deviation of $\mathrm{N}$ random networks.

3.11. Network Randomization. Network randomization is used to generate a random network for network comparison. During the randomization process, self-connection and duplication of edges are prohibited.

\section{Data Description and Processing}

4.1. Data Description. This dataset contains information on the operation status of all flights departing and arriving in China from December 1st to 31st 2000. The calculation method of the flight arrival delay in this article is the difference between the actual arrival time and the planned arrival time, representing the actual delay perceived by the passengers, rather than starting to calculate the arrival delay more than 15 minutes after the scheduled arrival time. We obtained the average daily arrival delay time for all flights in December 2000 across the country based on this calculation method. The results showed that the delay time on December $4,8,20$, and 21 was relatively high, while on the 11th and 17 th, the delays on the day and the 31st are relatively low as shown in Figure 1.

\subsection{Data Preprocessing}

4.2.1. Data Cleaning. Specific strong values need to be calculated based on the original data, such as flight arrival delay, departure delay, ground transit, and air flight. At the same time, there is a certain percentage of dirty data in the original dataset, which needs to be predicted. The detailed steps of preprocessing are as follows:

Step 1: delete 11 pieces of data whose planned arrival times are empty.

Step 2: 1649 entries.

Step 3: the original data only have the planned departure date and the actual departure date but not the planned arrival date and actual arrival date. To facilitate subsequent processing, the scheduled arrival date and actual arrival date need to be added to the data.

Step 4: for the convenience of subsequent processing, splice the two fields of date and time into one area.

Step 5: the original data are not the data of the flight segment level, so it is necessary to set whether each data are the tag of the flight segment, which is seg_count. It 
is not a flight segment level. For example, there is an aircraft flying from $A$ to $B$ and from $B$ to $C$, but there is another piece of data in the data that will pass from $A$ to C. Therefore, the seg_count field corresponding to the data flying from $\mathrm{A}$ to $\mathrm{C}$ is set to 2 . The seg_count flying from $A$ to $B$ and from $B$ to $C$ are both set to 1 . After the final setting is completed, there are 54891 data with seg_count $>1$.

Step 6: delete the data of seg_count $>1$, these data are not of the flight segment level, so they are useless.

Step 7: the flight registration number is the same, the departure airport is the same, the planned departure time is the same, and the arrival airport may have the same or different data. Only one of these data need to be deleted, but which one do you choose to delete? or delete one randomly (currently select the one with the smallest id), about 180.

Step 8: calculate the planned flight time and actual flight time (flight time $=$ arrival time-departure time) in minutes.

Step 9: calculate the planned passing time and the actual passing time. This month, this task needs to find the flight chain based on the aircraft registration number. The transit time $=$ the departure time of the next flight in the flight chain-the departure time of the previous flight in the flight chain, in minutes. Although this calculation does not consider the midway rest of some aircraft, there may be some very long transit times, but this does not affect the subsequent operations, so there is no need to worry.

Step 10: calculate take-off delay and arrival delay. Departure delay $=$ actual departure time-planned departure time, and arrival delay $=$ actual arrival timeplanned arrival time, in minutes.

Step 11: delete the data with the larger absolute value of the difference between the actual flight time and the planned flight time (currently take the data with the total value greater than 5 hours). From practical considerations, the flight time between the two places should not fluctuate too much, so the data that fluctuate more than 5 hours are deleted, 108 pieces. Of course, it is not necessarily correct if it is less than 5 hours, but there is no way.

Step 12: delete the data whose planned and actual transit time and flight time are less than zero, 3810 items.

Step 13: complete data preprocessing.

From the above 13 steps, all the fundamental values that need to be used can be obtained, the dirty data can be removed, and finally, 256758 flight operating status data can be obtained.

4.2.2. Construction of Airport Delay Data. The input for transferring entropy needs to be time-series data, so after data calculation and data cleaning, they still need further processing.

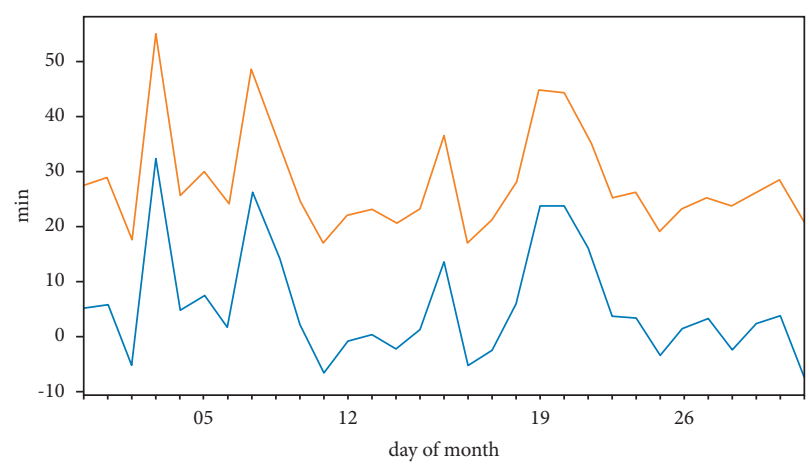

FIgURE 1: National average daily delay time.

Step 1. Aggregate the arrival delays of all flights at all airports at the hourly level to obtain the arrival delay time series of all airports.

Precisely, the average arrival delay time of all flights is calculated whose planned arrival time is within the $h$ hour on day $d$ at the $i$-th airport.

$$
D^{i}(d, h)=\frac{\sum\left(T_{a c t}-T_{\text {sch } d}\right)}{N^{i}(d, h)}, i \in\{1,2\},
$$

where $T_{\text {act }}$ represents the actual arrival time of all flights whose scheduled arrival time is within the $h$ hour on day $d$ of the $i$-th airport and $T_{\text {schd }}$ represents the actual arrival time of all flights whose scheduled arrival time is within the $h$ hour on day $d$ of the $i$-th airport. Planned arrival time, $N^{i}(d, h)$, represents the number of all flights whose planned arrival time is within the $h$ hour on the $d$ day of the i-th airport.

Then, the i-th airport arrival delay time-series set can be expressed as

$$
S^{i}=\left\{D^{i}(1,1), D^{i}(1,2), \ldots, D^{i}(2,1), D^{i}(2,2), \ldots, D^{i}(d, h)\right\} .
$$

Here, the length of the airport arrival delay time series is $n=d * h$.

Step 2. Use Z-score standardization to process the arrival delay time series, and the processing method is as follows:

$$
D^{i_{1}}(d, h)=\frac{D^{i}(d, h)-<D^{i}(\cdot, h)>}{\sigma\left(D^{i}(\cdot, h)\right)} \quad, i \in\{1,2\} .
$$

where $D^{i}(d, h)$ represents the normalized average arrival delay time of all planned arrival times at the $h$ th hour at the dth day of the i-th airport, $D^{i}(d, h)$ represents the original average arrival delay time of all planned arrival times at the $h$ th hour of the i-th airport on the $d$ day, $\left\langle D^{i}(\cdot, h)>\right.$ represents the average value of all sample points at the $h$-th hour of the $i$-th airport, and $\sigma\left(D^{i}(\cdot, H)\right)$ represents the standard deviation of all sample points at the h-th hour of the i-th airport.

Then, the preprocessed set of the i-th airport arrival delay time series can be expressed as 
$S^{i_{\prime}}=\left\{D^{i_{\prime}}(1,1), D^{i_{\prime}}(1,2), \ldots, D^{i_{\prime}}(2,1), D^{i_{\prime}}(2,2), \ldots, D^{i_{\prime}}(d, h)\right\}$.

Here, the length of the preprocessed airport arrival delay time series is $n=d * h$.

From the above two steps, the final required transfer entropy input time-series data can be obtained. The processing of the original data is completed.

\section{Experiment Analysis}

5.1. Global Characteristics of the Airport Delay Propagation Relationship Network. To analyze the delay propagation relationship, we need to transfer entropy between the paired computer field delay time series and build a network of delay propagation. We aggregate flight data hourly so that each airport corresponds to a time series every day. Then, we build a network of delay propagation relationships every day, and we get a total of 31 networks. December 4th is the day with the most serious flight delays. We use the communication network on this day to analyze the characteristics of delayed propagation. After removing the unconnected airports, it is found that there are 169 airport nodes and 1381 edges, as shown in Figure 2, which means that about three-quarters of the airports have a delayed propagation relationship with other airports that day. This delayed propagation relationship network includes $174 \mathrm{~F}-$ class airports, $354 \mathrm{E}$-class airports, 38 4D-class airports, 71 $4 \mathrm{C}$ airports, and eight $3 \mathrm{C}$ airports. It can be seen that larger airports such as $4 \mathrm{~F}, 4 \mathrm{E}$, and $4 \mathrm{D}$ are almost all caught in the spread of delays. Although small airports such as $4 \mathrm{C}$ and $3 \mathrm{C}$ occupy a large proportion of the entire civil aviation network, only half of them are involved in the spread of flight delays.

For this communication network, we will use network analysis tools to answer the following questions about delayed communication:

How many airports are affected or affected by each airport? For a certain airport $i$ in the network, $k_{\mathrm{i}}^{\text {out }}$ is the number of airports affected by airport $i$, and $k_{i}^{i n}$ is the number of airports affected by airport $i$. In this network, $\left\langle k^{\text {in }}\right\rangle=\left\langle k^{\text {out }}\right\rangle=8.17$, which means that each airport affects about 8 airports and is affected by about 8 airports.

Is the delayed propagation connection between airport pairs bidirectional? The reciprocity parameter of this network is $R=0.77$. Randomly, 1000 networks with the same number of nodes and edges are generated, and their average reciprocity coefficient is found to be 0.049 , which is much lower than 0.77 . Therefore, the network is more symmetrical. One possible reason is that delays caused by two-way flights between airports are prone to two-way propagation.

How big is the aggregation trend between airports? The average aggregation coefficient of the entire network is 0.42 , which is much larger than the average aggregation coefficient of the random network $(=0.048)$, indicating that the airport network has a strong aggregation tendency.

Can the delay propagation between airports be divided into multiple subareas? The community detection algorithm is used to analyze it. Modularity is used to evaluate the strength of the network divided into communities. The greater the modularity value, the more obvious the community structure. The modularity value of this network is 0.168 , and the average modulus value of 1000 random networks is 0.205 . Therefore, there is no evidence that the delayed propagation on December 4 can clearly delineate the subregions.

What is the possible range of delay propagation? We use the largest connected cluster to represent the range of possible delay propagation. The member airports of the largest connected cluster are selected by the $k^{\text {out }}$ threshold so that the largest connected cluster contains a set of airports that have more influence on other airports. We define the threshold $k^{\text {out }}$ to be greater than the average out-degree of the network $k^{\text {out }}=8.17$, which means that the impact of the airport is greater than or equal to the other five airports. The largest connected cluster of the network contains the number of airports $M_{\mathrm{d}}=50$, indicating that the delay may spread among 50 airports.

\subsection{Time Characteristics of the Delay Propagation Relation-} ship Network. As time changes, the daily delay situation is different; how will the delay propagation network change? To answer this question, we evaluated the Pearson correlation coefficient between the daily network topology indicators of the delay propagation relationship network and the average daily arrival delays of all flights in December. It can be seen from Table 1 that the number of edges, average clustering coefficient, average degree, link density, and comatch coefficient are all highly correlated with the average daily arrival delay. At the same time, the correlations of other indicators are relatively low. According to the formula of average degree and link density, it can be said that these two indicators are directly proportional to the number of sides, so the number of sides, average degree, and link density can be analyzed together. This result means that the longer the national average daily delay to Hong Kong, the more edges in the delay propagation network, and vice versa.

The higher the clustering coefficient of the node, it means that the neighboring nodes of the node are more likely to be connected. It is easier to form a local area with the node as the starting point so that the delay will spread in it; on the contrary, if the clustering coefficient of the node is lower, the node's neighbor nodes are more inclined to connect to other nodes except their neighbors. The formed network is closer to a tree so that the delay will spread quickly in a larger area. Therefore, due to the large degree and the low aggregation coefficient, the large airport will produce a more extensive range of delay propagation. In contrast, in the small airport, the delay generated can only spread in a local area due to the low degree and the high aggregation coefficient. 


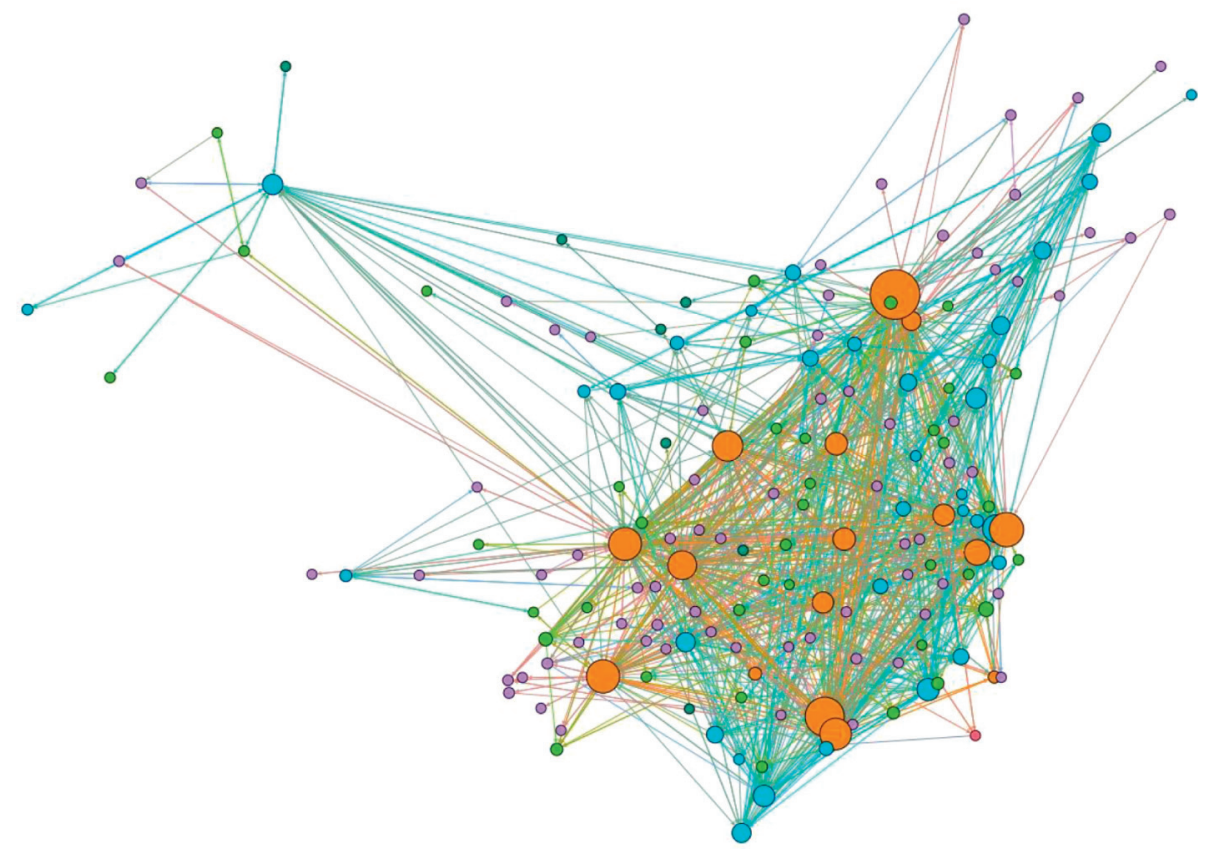

Figure 2: Delayed dissemination network on December 4.

TABLE 1: Correlation analysis between network topology indicators and average daily arrival delays.

\begin{tabular}{lc}
\hline Network topology indicators & Pearson correlation coefficient \\
\hline Number of nodes & 0.396 \\
Number of sides & 0.634 \\
Reciprocity coefficient & 0.550 \\
Average clustering coefficient & 0.652 \\
Average degree & 0.627 \\
Link density & 0.704 \\
Transitivity & 0.522 \\
Coefficient of coordination & -0.632 \\
Largest connected cluster & 0.327 \\
Modularity & -0.448 \\
Efficient & 0.457 \\
\hline
\end{tabular}

The co-match coefficient has an inverse relationship with the average daily arrival delay time, which means that the more serious the delay, the more nodes with higher degrees tend to nodes with lower degrees of connectivity, because the degree is proportional to the number of flights, and the number of flights is larger. The daily operation of the airport has approached saturation, so the more serious the delay, the greater the tendency of large airports to spread the delay to various small airports and try to avoid affecting other larger airports and reduce losses.

\section{Discussion and Conclusion}

From the perspective of the entire aviation network, this study established a delay propagation relationship network based on the time-series relationship of each airport arrival delay. It used network analysis and evaluation indicators to reveal the macro performance of delay propagation. Specifically, we have constructed 31 flight delay propagation relationship networks using the information on the operation status of all flights departing and arriving in China. Selecting the day with the most serious delays in the 31 days as the analysis object, we found that the larger airports of $4 \mathrm{~F}$, $4 \mathrm{E}$, and $4 \mathrm{D}$ are almost all caught in the spread of delays, although small airports such as $4 \mathrm{C}$ and 3C occupy a large proportion of the entire civil aviation network. But only half of them are involved in the spread of flight delays. The average degree shows that, on average, each airport affects about eight airports and is also affected by about eight airports. The very high reciprocity parameter indicates that the two-way delay spread between the airport pairs is more serious. It may be because a plane performs round-trip flights between the two airports a day, but there is no suitable buffer absorption mechanism between the airports to cause the delay. The high clustering coefficient of the airport indicates that the network has a strong trend of clustering, and the airport nodes tend to form a denser network cluster. We have also found the largest connected cluster in the network, where each airport node has more influence. The ingress and egress of airport nodes are directly proportional to the number of flights at the airport. The intensive flight of large airports has made the airport capacity close to saturation. Once an accident occurs, the delay will happen and spread, but because large airports generally have better-delayed handling measures may reduce some of the delays.

Next, we analyzed the time characteristics of the delay propagation relationship network, compared 31 indicators of the delay propagation relationship network, and calculated the Pearson correlation coefficient between each indicator and the average daily arrival delay of all flights in December. The results obtained show that the aggregation coefficient strongly correlates positively with the average daily arrival delay. This explains that large airports have a 
larger degree and a low aggregation coefficient, which will cause a larger range of delay propagation, while small airports have a higher degree of delay. Small and high aggregation coefficients and the resulting delay can only spread in a local area. The co-match coefficient has an inverse relationship with the average daily arrival delay time. This means that the more serious the delay, the more nodes with higher degrees tend to nodes with lower degrees of connectivity. Therefore, the more serious the delay, the greater the tendency for large airports to spread the delay. Delays can be dispersed to various small airports to avoid affecting other larger airports and reduce losses. The proposed method is a general causal analysis method. In the future, we will use this method on other types of time-series data, such as physiological time series and financial time series.

\section{Data Availability}

The dataset can be accessed upon request to the corresponding author.

\section{Conflicts of Interest}

The authors declare that they have no conflicts of interest.

\section{Acknowledgments}

This study was supported by the University industry university research innovation fund of science and technology development center of the Ministry of education of China (2020ITA05022), the Natural Science Foundation of Hubei Province (2021CFB316), the Preliminary Support Project of Hubei Social Science Foundation (21ZD137), and the Hundreds of Schools Unite with Hundreds of CountiesUniversity Serving Rural Revitalization Science and Technology Support Action Plan (BXLBX0847).

\section{References}

[1] John Wiley \& Sons, The Global Airline Industry, John Wiley \& Sons, Hoboken, NJ, USA, 2015.

[2] M. Ball, C. Barnhart, M. Dresner et al., Total Delay Impact study, NEXTOR Research Symposium, Washington DC, USA, 2010, http://www.nextor.org.2010.

[3] S. AhmadBeygi, A. Cohn, Y. Guan, and P. Belobaba, "Analysis of the potential for delay propagation in passenger airline networks," Journal of Air Transport Management, vol. 14, no. 5, pp. 221-236, 2008.

[4] A. I. Czerny, "Airport congestion management under uncertainty," Transportation Research Part B: Methodological, vol. 44, no. 3, pp. 371-380, 2010.

[5] N. Kafle and B. Zou, "Modeling flight delay propagation: a new analytical-econometric approach," Transportation Research Part B: Methodological, vol. 93, pp. 520-542, 2016.

[6] S. Lan, J.-P. Clarke, and C. Barnhart, "Planning for robust airline operations: optimizing aircraft routings and flight departure times to minimize passenger disruptions," Transportation Science, vol. 40, no. 1, pp. 15-28, 2006.

[7] L. Daqing, J. Yinan, K. Rui, and S. Havlin, "Spatial correlation analysis of cascading failures: congestions and Blackouts," Scientific Reports, vol. 4, no. 1, p. 5381, 2014.
[8] L. Meng and X. Zhou, "Robust single-track train dispatching model under a dynamic and stochastic environment: a scenario-based rolling horizon solution approach," Transportation Research Part B: Methodological, vol. 45, no. 7, pp. 1080-1102, 2011.

[9] R. Beatty, R. Hsu, L. Berry, and J. Rome, "Preliminary evaluation of flight delay propagation through an airline schedule," Air Traffic Control Quarterly, vol. 7, no. 4, pp. 259-270, 1999.

[10] L. W. Fan, F. Wu, and P. Zhou, "Efficiency measurement of Chinese airports with flight delays by directional distance function," Journal of Air Transport Management, vol. 34, pp. 140-145, 2014.

[11] W.-B. Du, M.-Y. Zhang, Y. Zhang, X.-B. Cao, and J. Zhang, "Delay causality network in air transport systems," Transportation Research Part E: Logistics and Transportation Review, vol. 118, pp. 466-476, 2018.

[12] M. Maziarz, "A review of the Granger-causality fallacy," The Journal of Philosophical Economics: Reflections on economic and social issues, vol. 8, no. 2, pp. 86-105, 2015.

[13] G. Ver Steeg and A. Galstyan, "Information transfer in social media," in Proceedings of the 21st international conference on World Wide Web, pp. 509-518, New York, United States, 16 April 2012.

[14] M. Lungarella, K. Ishiguro, Y. Kuniyoshi, and N. Otsu, "Methods for quantifying the causal structure of bivariate time series," International journal of bifurcation and chaos, vol. 17, no. 3, pp. 903-921, 2007.

[15] N. Pyrgiotis, K. M. Malone, and A. Odoni, "Modelling delay propagation within an airport network," Transportation Research Part C: Emerging Technologies, vol. 27, pp. 60-75.

[16] N. Nayak and Y. Zhang, "Estimation and comparison of impact of single airport delay on national airspace system with multivariate simultaneous models," Transportation Research Record: Journal of the Transportation Research Board, vol. 2206, no. 1, pp. 52-60, 2011.

[17] L. Hao, M. Hansen, Y. Zhang, and J. Post, "New York, New York: two ways of estimating the delay impact of New York airports," Transportation Research Part E: Logistics and Transportation Review, vol. 70, pp. 245-260, 2014.

[18] Z. Jia, Y. Lin, Y. Liu, Z Jiao, and J Wang, "Refined nonuniform embedding for coupling detection in multivariate time series," Physical Review, vol. 101, no. 6, Article ID 062113, 2020.

[19] P. Fleurquin, J. J. Ramasco, and V. M. Eguiluz, "Systemic delay propagation in the US airport network," Scientific Reports, vol. 3, no. 1, p. 1159, 2013.

[20] B. Campanelli, P. Fleurquin, A. Arranz et al., "Comparing the modeling of delay propagation in the US and European air traffic networks," Journal of Air Transport Management, vol. 56, pp. 12-18, 2016.

[21] Z. Jia, Y. Lin, Z. Jiao, Y. Ma, and J. Wang, "Detecting causality in multivariate time series via non-uniform embedding," Entropy, vol. 21, no. 12, p. 1233, 2019.

[22] Z. Jia, Y. Lin, J. Wang, Z. Feng, X. Xie, and C. Chen, "HetEmotionNet: two-stream heterogeneous graph recurrent neural network for multi-modal emotion recognition," 2021, http://arXiv.org/abs/2108.03354.

[23] Z. Li, J. Wang, Z. Jia, and Y. Lin, "Learning space-time-frequency representation with two-stream attention based 3D network for motor imagery classification," in Proceedings of the 2020 IEEE International Conference on Data Mining (ICDM), pp. 1124-1129, IEEE, Sorrento, Italy, 17-20 Nov. 2020. 
[24] Z. Jia, Y. Lin, T. Liu, K. Yang, X. Zhang, and W. Jing, "Motor imagery classification based on multiscale feature extraction and squeeze-excitation model," Journal of Computer Research and Development, vol. 57, no. 12, p. 2481.

[25] Z. Jia, Y. Lin, X. Cai, H. Chen, H. Gou, and J. Wang, "Sstemotionnet: spatial-spectral-temporal based attention $3 \mathrm{~d}$ dense network for eeg emotion recognition," in Proceedings of the 28th ACM International Conference on Multimedia, pp. 2909-2917, WA, Seattle, USA, 12 October 2020.

[26] Z. Jia, Y. Lin, J. Wang, K. Yang, T. Liu, and X. Zhang, “A multi-branch multi-scale convolutional neural network for motor imagery classification," in Proceedings of the Joint European Conference on Machine Learning and Knowledge Discovery in Databases, pp. 736-751, Springer, Ghent, Belgium, 14-18 September.

[27] Y. Liu, Y. Lin, Z. Jia, J. Wang, and Y. Ma, “A new dissimilarity measure based on ordinal pattern for analyzing physiological signals," Physica A: Statistical Mechanics and Its Applications, vol. 574, Article ID 125997, 2021.

[28] Y. Liu, Y. Lin, Z. Jia, Y. Ma, and J. Wang, "Representation based on ordinal patterns for seizure detection in EEG signals," Computers in Biology and Medicine, vol. 126, Article ID 104033, 2020.

[29] Z. Jia, Y. Lin, J. Wang, R. Zhou, and X. Ning, "GraphSleepNet: adaptive spatial-temporal graph convolutional networks for sleep stage classification," IJCAI, pp. 1324-1330, 2020.

[30] Z. Jia, Y. Lin, J. Wang, X. Wang, P. Xie, and Y. Zhang, "SalientSleepNet: multimodal salient wave detection network for sleep staging," arXiv preprint: http://arXiv.org/abs/2105. 13864, 2021.

[31] X. Cai, Z. Jia, M. Tang, and G. Zheng, "Brainsleepnet: learning multivariate eeg representation for automatic sleep staging," in Proceedings of the IEEE International Conference on Bioinformatics and Biomedicine, pp. 976-979, Seoul, Korea, 16-19 Dec. 2020.

[32] Z. Jia, X. Cai, G. Zheng, J. Wang, and Y. Lin, "SleepPrintNet: a multivariate multimodal neural network based on physiological time-series for automatic sleep staging," IEEE Transactions on Artificial Intelligence, vol. 1, no. 3, pp. 248-257, 2020.

[33] Z. Jia, Y. Lin, H. Zhang, and J. Wang, "Sleep stage classification model based ondeep convolutional neural network," Journal of Zhejiang University (Science Edition), vol. 54, no. 10, pp. 1899-1905, 2020.

[34] Z. Jia, Y. Lin, J. Wang et al., "Multi-view spatial-temporal graph convolutional networks with domain generalization for sleep stage classification," IEEE Transactions on. Neural Systems and Rehabilitation Engineering, vol. 29, pp. 970-979, 2021.

[35] W. Li, Q. A. Wang, L. Nivanen, and A. Le Méhauté, "How to fit the degree distribution of the air network?" Physica A: Statistical Mechanics and Its Applications, vol. 368, no. 1, pp. 262-272, 2006

[36] G. Bagler, "Analysis of the airport network of India as a complex weighted network," Physica A: Statistical Mechanics and Its Applications, vol. 387, no. 12, pp. 2972-2980, 2008.

[37] R. Guimera, S. Mossa, A. Turtschi, and L. A. N. Amaral, "The worldwide air transportation network: anomalous centrality, community structure, and cities' global roles," Proceedings of the National Academy of Sciences, vol. 102, no. 22, pp. 7794-7799, 2005.

[38] Z.-C. Li, W. H. K. Lam, S. C. Wong, and X. Fu, "Optimal route allocation in a liberalizing airline market," Transportation
Research Part B: Methodological, vol. 44, no. 7, pp. 886-902, 2010.

[39] H. E. Silva, E. T. Verhoef, and V. A. C. Van den Berg, "Airline route structure competition and network policy," Transportation Research Part B: Methodological, vol. 67, pp. 320343, 2014.

[40] D. Gillen, H. Hasheminia, and C. Jiang, "Strategic considerations behind the network-regional airline tie ups-a theoretical and empirical study," Transportation Research Part B: Methodological, vol. 72, pp. 93-111, 2015. 